\title{
El empleo de modelos metaheurísticos en la logística industrial. El caso del enrutamiento de vehículos
}

Orestes Cachay Boza ${ }^{1}$ Eduardo Raffo Lecca ${ }^{2}$ Adolfo Acevedo Borrego ${ }^{3}$

\section{RESUMEN}

El problema del enrutamiento de vehículos (Vehicle Routing Problem) considera el diseño de conjuntos de rutas a costo mínimo, originados y terminados desde y hacia un punto denominado depósito. En esta situación problemática, un elemento clave en los sistemas de suministro es la secuencia de las unidades de transporte, determinado por el requerimiento de los clientes.

Este artículo tiene el objetivo de plantear un nuevo método de solución al problema de asignación de enrutamiento de las empresas que tienen la necesidad de distribuir productos, mediante nuevos modelos que emplean diversos algoritmos. El modelo elaborado a partir del análisis de una serie de metaheurísticas, desarrolla opciones factibles para resolver el problema diario de cómo asignar y distribuir productos de la empresa hacia los clientes de manera eficiente y eficaz.

Palabras clave: Modelo, Metaheurística, Decisiones, Sistema de suministro.

\section{THE USE OF METAHEURISTICS} MODELS IN INDUSTRIAL LOGISTICS. THE CASE OF VEHICLE ROUTING

ABSTRACT

The Vehicle Routing Problem considers the design of sets of minimal cost routes, from an originated point knowned as deposit to an ended point. In this problematic situation, a key element in delivery systems is the sequencing of transport units, determined by the requirement of customers.

The aim of this article is to present a new method of solving the problem of allocation of routing companies with the need to distribute products, through new models that use different algorithms. The model designed from the analysis of a set of metaheuristics, develop viable options to solve the daily problem of how to allocate and distribute products, from the company to customers, with efficiently and effectively ways.

Keywords: Model, Metaheuristics, Decisions, Supply System.

\section{INTRODUCCIÓN}

El presente trabajo tiene el objetivo de mostrar un nuevo algoritmo para enfrentar el problema de delivery de producto, cual es conocido en la literatura como Vehicle Routing o VRP.

Para la resolución del problema de planificación de rutas de transporte se han desarrollado una amplia variedad de métodos y técnicas, desde exactas, hasta heurísticas y metaheurísticas. Los primeros requieren una elevada inversión en tiempo de procesamiento y cálculo, los segundos aportan soluciones aproximadas con la ventaja de emplear un reducido tiempo de procesamiento.

La creciente importancia de la logística empresarial, ha generado una mayor complejidad y profundización de algoritmos basados en procesos metaheurísticos (8) como los algoritmos genéticos, temple simulado, búsqueda Tabú, GRASP, Búsqueda Local Guiada (GLS), colonia de hormigas; desarrollados a partir de los trabajos de Gendreau (1991 y 1994), Osman (1993), recientemente el trabajo de Potvin y otros (1993), Thangiah (1993), Campos y Mota (1995), Kantoravdis (1995), Rochat (1995), Kilby (1997), Backer (1997), Bullnheimer (1997) y Rego (1998).

En el problema de optimización combinatoria se emplean algoritmos de búsqueda local (o búsqueda vecinal) dentro de las técnicas heurísticas, y algoritmos de búsqueda tabú, como procedimiento metaheurístico, cuyos antecedentes se encuentran en el trabajo de Glover (1977), también en los trabajos de Bodin y Golden (1981), Desrochers (1988), Haouri $(1,990)$, Laporte (1992) y Laporte y Osman (1995).

\section{ESTADO DEL ARTE EN EL PROBLEMA DE ENRUTAMIENTO}

El tema de enrutamiento representa un problema de asignación que recibe mayor atención en diferentes campos de la investigación empresarial, desde las matemáticas aplicadas, Investigación de operaciones y la metodología de sistemas flexibles (3). El enrutamiento comprende dos clases de problemas: cubrimiento de arcos (edge-covering) y cubrimiento de puntos o

\footnotetext{
Profesor en la Facultad de Ingeniería Industrial, Departamento de Producción y Gestión Industrial de la UNMSM. Email: orestescachay@yahoo.es

2 Profesor en la Facultad de Ingeniería Industrial, Departamento de Producción y Gestión Industrial de la UNMSM. Email: eraffolecca@yahoo.es

3 Profesor en la Facultad de Ingeniería Industrial, Departamento de Producción y Gestión Industrial de la UNMSM. Email: aacevedo@speedy.com.pe
} 
nodos (node-covering). En las entregas o delivery, recojo o pickup y visitas, se hace en un número específico de puntos: el problema del enrutamiento, deberá ser resuelto por el cubrimiento entre nodos o node-covering. Los puntos de demanda u oferta, pueden ser representados como puntos en un modelo de redes, como ocurre en una rejilla de un transporte urbano, donde la cuestión a resolver se refiere al orden de visitas a los nodos, a fin de cumplir un objetivo específico de eficiencia de distribución.

El problema del agente viajero o TSP (Traveling Salesman Problem), es el problema fundamental y más conocido en los casos node-covering. Se considera un cartero responsable de entregar la correspondencia en un área de la ciudad (15). El cartero debe siempre empezar su ruta de delivery en un punto $A$, donde se encuentra ubicada la oficina postal; deberá atravesar cada calle en su área, y deberá retornar al punto $A$. La longitud de cada arco o borde (edge), representa el segmento de la calle a visitar. La gráfica que resulta de la representación de las calles, con sus nodos o puntos de intersección entre las calles, origina una gráfica no dirigida, más conocida como grafo (sin dirección, por no existirla para el cartero). La ruta del cartero deberá ser diseñada para minimizar la distancia total que camina, restringida por el hecho que cada segmento de calle, solo deberá ser atravesada una vez.

En el TSP, cada nodo debe ser visitado exactamente una vez, sean $\mathrm{n}$ puntos en una red, y deberán visitarse los $n-1$ restantes, por un vehículo; el cual empieza y termina en un punto específico, denominado depósito (depot). La red de transporte que conecta a todos los puntos es una gráfica completamente conectada. La distancia más corta entre todos los pares de puntos es igual a la longitud de la conexión directa, entre dos pares de puntos; si $\boldsymbol{i}$ y $\boldsymbol{j}$ son dos puntos, de los $n$ puntos, entonces $\boldsymbol{d}(\boldsymbol{i}, \boldsymbol{j})=\boldsymbol{d}(\boldsymbol{i}, \boldsymbol{j})$. Esto implica que la red, satisface la desigualdad triangular: $\delta(i, j)=\delta(i, k)+\delta(k, j)$, para cada tres puntos $(\boldsymbol{i}, \boldsymbol{j}, \boldsymbol{k})$. Agréguese, que se asume que es simétrica la matriz de las distancias más cortas. La completa conectividad de la red, y la desigualdad triangular, aseguran que el tour del agente viajero, tiene la longitud más corta a través de los $n$ puntos; en donde cada punto es visitado solo una vez.

Existen una diversidad de variantes y extensiones al TSP correspondiendo al conjunto cubrimiento de nodos. De acuerdo a la clasificación node-covering, existen tres descripciones: a) el número de vehículos (vendedores), b) el número de tours de oríge- nes, c) existencia de restricciones, como capacidad individual del vehículo, longitud máxima del tiempo de una ruta. Otros problemas, sin las restricciones anteriores, son variaciones TSP; la ruta es realizada por un vendedor o un vehículo, usando un origen-destino simple. El problema del m-TSP, envuelve el diseño de un número preconcebido $m$, de distintas rutas, que colecciona la demanda de los puntos en cada visita, usando un punto común (o varios) orígenes-destinos. El objetivo es minimizar la distancia total cubierta en $m$ rutas.

\section{TÉCNICAS PRINCIPALES PARA LA SOLUCIÓN VRP}

El problema del enrutamiento de vehículos o VRP (Vehicle Route Problem) se manifiesta cuando existen las restricciones en la capacidad de los vehículos (17). Las técnicas para su solución son:

- Optimización, considerando una jerarquía de problemas y su correspondiente colección de técnicas de solución (14).

- Complejidad computacional, esta se enfoca en el costo de la resolución del problema (12).

- Heurística o procedimiento simple, es basado en el sentido común que ofrece una buena solución a problemas difíciles de un modo fácil y rápido.

- Metaheurística o procedimiento maestro de alto nivel, que guía y modifica otras heurísticas para explorar soluciones más allá de la simple optimalidad local.

\section{a) Optimización}

Estos problemas, son conocidos como el problema de la programación no lineal: donde se encuentra el valor de $x$, tal que:

$$
\min f\left(x_{1}, x_{2}, \ldots, x_{n}\right)
$$

sujeto a

$$
\begin{array}{ll}
g_{i}\left(x_{1}, x_{2}, \ldots, x_{n}\right) \geqq b_{i}, & i=1, \ldots, m \\
h_{j}(x)=0, & j=1, \ldots, p
\end{array}
$$

Donde $f, g$ y $h$ son funciones generales del parámetro $x \in R^{n}$. Las técnicas para resolver tales problemas son siempre iterativas por naturaleza y su convergencia es estudiada usando matemáticas del mundo real. 
Si tanto restricciones, como función objetivo son lineales (Programación Lineal o PL), la existencia de máximo (mínimo) está asegurada, y el problema se reduce a la aplicación de unos simples algoritmos de álgebra lineal elemental, los llamado método simplex y dual. Sin embargo, si estas condiciones no se cumplen, existen las llamadas condiciones de Khun-Tucker, las cuales en algunos casos pueden ser utilizables para encontrar puntos críticos máximos o mínimos. Sin embargo, esta es un área aún muy poco desarrollada de la matemática frecuentemente, las condiciones de Khun-Tucker fallan o no son suficientes para la existencia de extremos. Cuando las variables del problema (función objetivo y/o restricciones) son variables aleatorias, el tipo de optimización realizada es denominada optimización estocástica. Ver Figura 1.

Figura 1. Clases de problemas en programación matemática

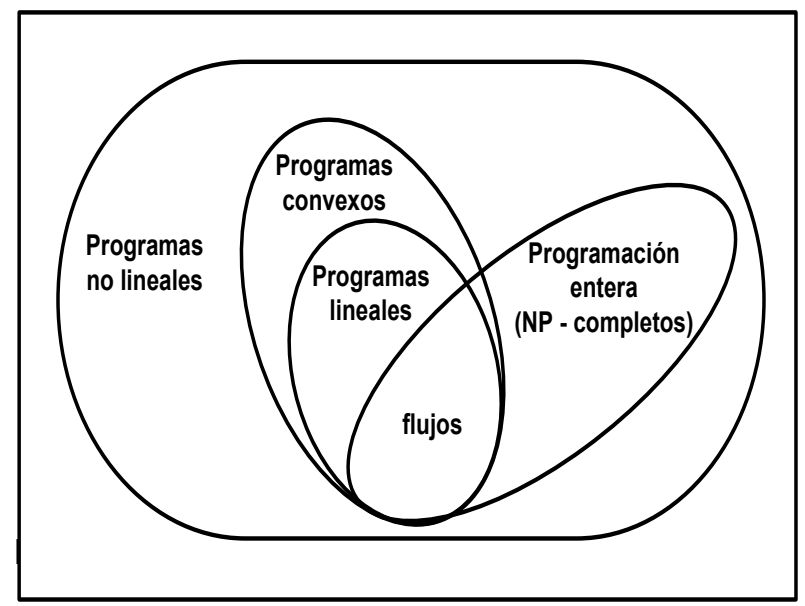

Geométricamente, los programas no lineales, se diferencian de un programa lineal. Suponga que la Figura 2 ilustra un programa no lineal. Se quiere minimizar una función $f(x)$, en el intervalo con $0 \leq x \leq 8$. El punto $x=5$ es el óptimo. El punto $x=0$, es una buena solución factible y es una solución óptima en la vecindad de $x=0$.

Aquí se distinguen, dos tipos de soluciones un óptimo local está en un lado, y un punto global en el otro lado, el punto de $x=5$. Un óptimo local es un óptimo con respecto a la solución factible en una región cerrada en dicho punto.
Figura 2: Óptimos locales y globales

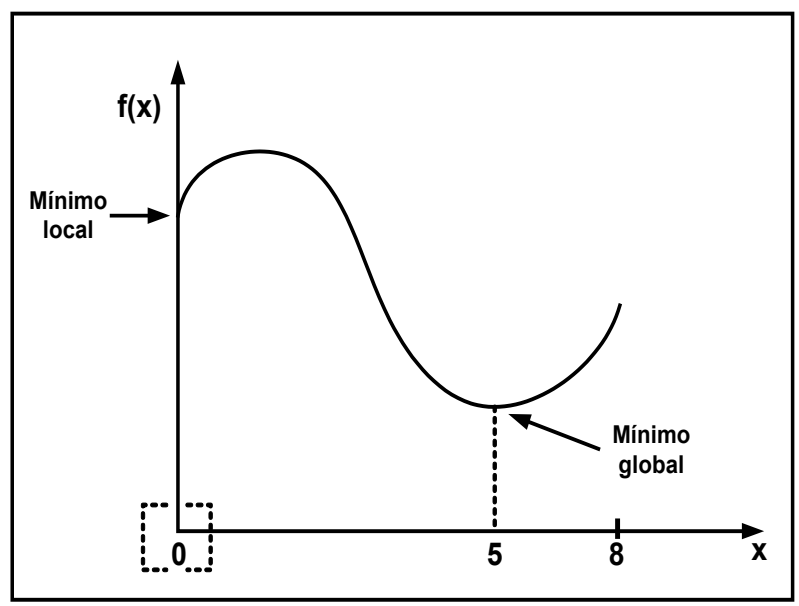

\section{b) Complejidad computacional}

Ante un problema concreto, existe una serie de algoritmos que se pueden aplicar. Cuando se resuelve un problema, se busca la mejor solución entre un conjunto de posibles soluciones. Al conjunto de todas las posibles soluciones a un problema concreto se llama espacio de búsqueda. El buscar una solución, se reduce a buscar un valor extremo (mínimo o máximo) en el espacio de búsqueda, aunque a veces el espacio de búsqueda puede ser bien definido, pero en la mayoría de las ocasiones solo se conocen algunos puntos en el espacio de búsqueda. Los problemas de esta clase se denominan NP (la N de no-deterministas y la $\mathrm{P}$ de polinómicos).

Existen problemas resolubles de manera determinista mediante algoritmos polinómicos en un tiempo polinomial, por ejemplo, la resolución de ecuaciones, la realización de sumas, productos. Estos son los problemas $\mathbf{P}$. Los algoritmos de complejidad polinómica, se dice que son tratables, en el sentido de que suelen ser abordables en la práctica. Los problemas para los que se conocen algoritmos con esta complejidad se dice que forman la clase $\mathbf{P}$.

También existen problemas NP que se pueden resolver de forma no determinista, probando una solución conjeturada. Esta comprobación es de una gran rapidez en comparación con el tiempo polinomial necesario en general para la resolución determinista de los problemas $\mathbf{P}$.

\section{c) Heurística}

Un método heurístico es un procedimiento que trata de descubrir una solución factible, pero no necesariamente óptima. Estos métodos se justifican por la existencia de problemas de optimización perte- 
necientes a la categoría denominada NP. Es decir, problemas para los que no existe un algoritmo de resolución que sea polinomial con el tamaño del problema. Si se demuestra que un problema de optimización pertenece a esta categoría de problemas NP, es práctica habitual el abordarlo por medios heurísticos de optimización. Se emplea en los problemas donde la búsqueda exhaustiva se torna ineficiente o en los problemas donde la cardinalidad del espacio de búsqueda aumenta exponencialmente con el tamaño del problema.

\section{d) Métodos Metaheurísticos}

Una metaheurística es un método de solución general, que proporciona tanto una estructura general, como criterios estratégicos para desarrollar un método específico que se ajuste a un tipo particular del problema (4). La Tabla 1 muestra las técnicas metaheurísticas más empleadas en la resolución de problemas.

Tabla 1. Principales técnicas metaheurísticas

\begin{tabular}{|l|l|}
\hline Nombre & \multicolumn{1}{|c|}{ Descripción } \\
\hline ACO & Optimización por colonias de hormigas \\
\hline AMP & Programas de memoria adaptativa \\
\hline CA & Algoritmos culturales \\
\hline EA & Algoritmos evolutivos \\
\hline FANS & Búsqueda por entorno adaptativo borroso \\
\hline GA & Algoritmos genéticos \\
\hline GHC & Métodos de escalada generalizados \\
\hline GLS & Búsqueda local guiada \\
\hline GRASP & $\begin{array}{l}\text { Procedimientos de búsqueda miope, aleatorizada y } \\
\text { adaptativa }\end{array}$ \\
\hline ILS & Búsqueda local iterativa \\
\hline MA & Algoritmos meméticos \\
\hline NM & Métodos ruidosos \\
\hline PR & Re-encadenamientos de caminos \\
\hline SA & Recocido simulado \\
\hline SI & Inteligencia de enjambre \\
\hline SS & Búsqueda dispersa \\
\hline TAM & Métodos de aceptación de umbral \\
\hline TS & Búsqueda Tabú \\
\hline VNS & Búsqueda de entorno variable \\
\hline
\end{tabular}

Existen heurísticas trayectoriales y poblacionales. Entre las metaheurísticas trayectoriales destacan las basadas en búsqueda local (como TS y SA), búsqueda iterativa (como ILS y FANS) y búsqueda multi-arranque (como GRASP). Por otro lado, en- tre las metaheuristicas poblacionales, destacan las basadas en combinación de soluciones (como GA y SS) y las basadas en movimientos (como ACO, de optimización de colonias de hormigas y SI, inteligencia de enjambres).

\section{EL CASO DE ENRUTAMIENTO DE VEHÍCULOS EN UNA EMPRESA DE DISTRIBUCIÓN}

Uno de los más importantes problemas en el sistema de transporte es el diseño de rutas para vehículos o personas (7). En algunos casos, estas rutas deben de ser diseñadas para que ellas atraviesen un camino exhaustivo de calles en una vecindad (2) o en alguna parte específica de una ciudad, u ocasionalmente en toda la ciudad.

La empresa OLDR S.A. cuenta con 6 años de funcionamiento, aunque la experiencia en la comercialización y distribución de combustibles viene desde 16 años atrás. Se estima un crecimiento del 10\% anual en las estaciones de atención.

\section{Las operaciones de la empresa}

Las operaciones en OLDR S.A., comprenden:

- Una red de estaciones de servicios propias operadas por OLDR.

- La ubicación es en la ciudad de Lima.

- El stock es de propiedad de OLDR hasta la descarga en cada punto.

- Describir los pasos del proceso estratégico orientados a lograr una presencia competitiva.

- Describir cómo se aplica la teoría de los sistemas en las operaciones.

- Explicar se logra mantener el nivel competitivo de la organización con productos de alta calidad o bajo costo, con un sistema de distribución con el mejor uso de recursos, costos e inversión, satisfacción oportuna de la demanda de la clientela, flexibilidad de oferta a través de la tecnología.

- Analizar el mejor uso de la capacidad para mejorar el nivel de distribución.

- Enfocar la gestión de la capacidad flexibilidad y planeamiento.

- Relacionar la estrategia de operaciones con la cadena de suministros.

- Contempla la planificación de la necesidad y la flota. 
Este conjunto de decisiones se enmarca en la Cadena de Suministro de la empresa.

\section{Restricciones}

Existen una serie de restricciones como horario y capacidad:

- Por horario: Se ha de considerar la disponibilidad de recepción de todas las estaciones.

- Por cisterna: Se debe considerar que no todas las estaciones reciben unidades de la misma capacidad por diversos factores, pudiendo ser por área de la estación, restricciones municipales u otros. Se tienen que enviar unidades de acuerdo a la necesidad de la estación. Por ejemplo, algunas solo pueden recibir unidades de 3,000 a 4,000 gls. como máximo.

Las cisternas pueden tener un solo compartimiento así como también varios compartimientos, dependiendo del volumen a programar se debe cuadrar con cada compartimiento.

Se considera el volumen a programar de acuerdo a la necesidad de cada estación, al trabajar con el método del pre-planning se conoce:

- La capacidad de tanques de los diferentes productos de cada estación.

- El stock inicial de las 7 am de cada estación por varilla, ya que estos datos se ingresan al sistema y programación y se los visualiza por medio de un reporte.

- Las ventas diarias de cada estación.

- El volumen muerto de cada estación.

- El volumen máximo a enviar a cada estación.

Para el ruteo de las unidades se considera que las estaciones estén ubicadas geográficamente cerca para optimizar tiempos y costos. Para lograr el objetivo, se tiene un programa para visualizar de una manera más amplia las estaciones, sus ubicaciones y restricciones. A esto se agrega, como un factor importante, la experiencia y buen criterio del área de programación para cumplir con el total de la demanda en el tiempo establecido.

En la programación de los viajes se debe considerar el tiempo de recorrido y se le agrega el tiempo operativo en planta como es el de carga y precintado. La logística de distribución de combustible, a 27 estaciones de servicios OLDR, a través del sistema de pre-planning. (Ver Figura 3).
Figura 3. Mapa de distribución de la empresa

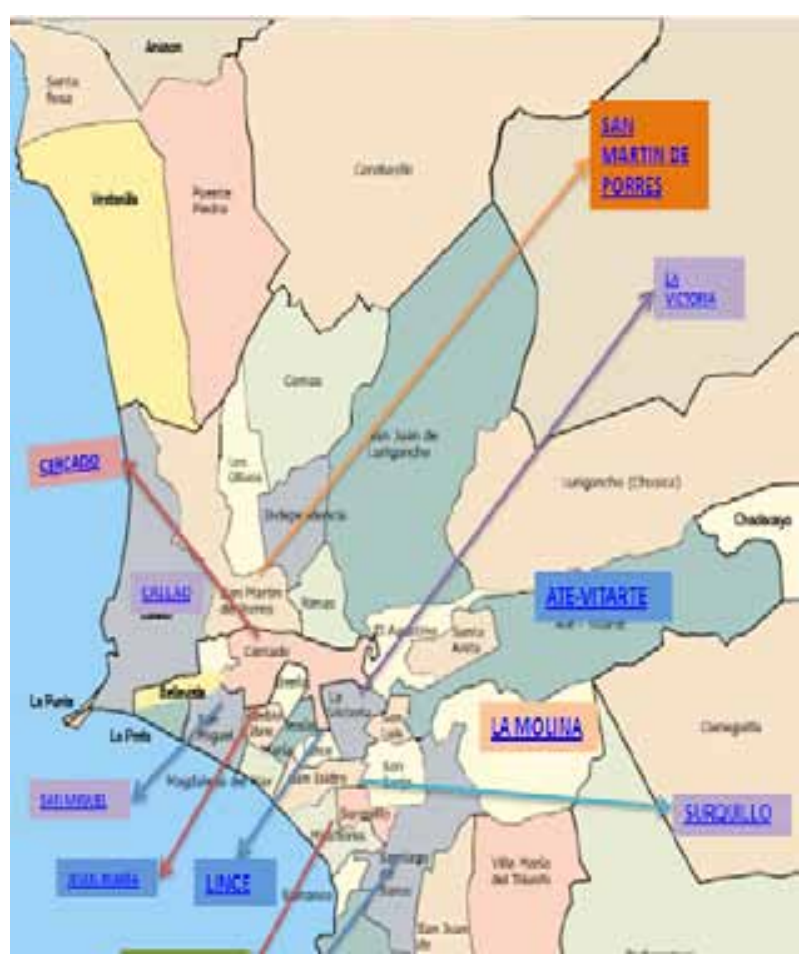

\section{Características del caso}

En el VRP clásico, un conjunto de clientes y un depósito ubicados geográficamente en puntos muy dispersos con una flota de vehículos; requieren de un plan de entrega de los bienes con la finalidad de minimizar sus costos, los que comienzan en el depósito y terminan en el mismo tal que un cliente es visitado una sola vez y no se exceda la capacidad de cada vehículo de la flota.

El cliente tiene una demanda determinista que debe ser recogida por el vehículo. En este punto que sea entrega o recojo vale para los mismos fines. La demanda es un bien que ocupa un lugar en el vehículo, y como un mismo vehículo no puede satisfacer a todos clientes, se hace necesario contar con una flota de vehículos, tal que la suma de los bienes que lleva el vehículo no deberá de exceder a su capacidad. En la literatura este es el problema de la mochila o Knapsack Problem (5). El hecho de que el vehículo se encuentre capacitado, impone una cota sobre la cantidad de clientes que puede satisfacer.

Se exige que cada ruta, comience y finalice en un punto denominado depósito. Para el caso de la logística, comúnmente, este es un lugar denominado almacén. Para el transporte de combustible esto significa la refinería, donde el vehículo cisterna recoge el combustible para ser distribuido. 
Existen modelos VRP que en contraposición al CVRP o VRP clásico, disponen de restricciones en los tiempos de entrega, son los llamados VRP con ventanas de tiempo (VRP with Time Windows) o VRPTW. En la actualidad, con las reglamentaciones locales o regionales en materia ambiental, exigen que se respeten los intervalos de tiempos en los cuales no es posible realizar operaciones de transporte o entrega (16); por el tema también de la seguridad.

Con respecto a los vehículos encargados del transporte, comúnmente todos tienen la misma capacidad, denominándose capacidad homogénea, en el caso contrario son de capacidad heterogénea. El número de unidades de vehículos en la flota es irrelevante cuando se tiene como objetivo la minimización del costo del transporte o su equivalente el total de recorrido. Existen situaciones en que se quiere el tamaño de la flota y esta se convierte en una decisión a resolver.

\section{LA APLICACIÓN DEL ALGORITMO DE SOLU- CIÓN DEL VRP}

\section{Recolección de datos}

La empresa OLDR es una de las empresas, que comparten con un gran competidor y otras menores, la venta de combustibles, a través de su red de grifos, en la ciudad de Lima.

La empresa OLDR es una de las empresas reconocidas y de mayor poder de mercado en el negocio de la venta de combustible.

La localización de los puntos de venta para las necesidades de un día típico se muestra en la Tabla 2.

Tabla 2. Necesidad de demanda en un día

\begin{tabular}{|c|l|c|c|c|c|}
\hline Orden & \multicolumn{1}{|c|}{ Estaciones } & $\begin{array}{c}\text { Stock de } \\
\text { Reposición }\end{array}$ & $\begin{array}{c}\text { Venta } \\
\text { Promedio }\end{array}$ & $\begin{array}{c}\text { Capacidad } \\
\text { Nominal }\end{array}$ & $\begin{array}{c}\text { Capa- } \\
\text { cidad } \\
\text { Real }\end{array}$ \\
\hline 1 & Sede (punto de origen) & 0 & 0 & 0 & 0 \\
\hline 2 & Sudamericano & 1700 & 500 & 2500 & 2,125 \\
\hline 3 & Carmelo & 1100 & 800 & 2500 & 2,125 \\
\hline 4 & Huiracocha & 1700 & 800 & 3000 & 2,550 \\
\hline 5 & Monterrico & 1700 & 800 & 3000 & 2,550 \\
\hline 6 & Canadá & 1700 & 800 & 3000 & 2,550 \\
\hline 7 & Igarza & 1700 & 600 & 3000 & 2,550 \\
\hline 8 & Quilca & 1700 & 800 & 3000 & 2,550 \\
\hline 9 & Zarate & 1700 & 1000 & 3000 & 2,550 \\
\hline 10 & La Paz & 1700 & 400 & 3000 & 2,550 \\
\hline 11 & La Marina - ACOSA & 1700 & 1500 & 3000 & 2,550 \\
\hline
\end{tabular}

\begin{tabular}{|c|l|c|c|c|c|}
\hline 12 & Ejército - Acosa & 1100 & 450 & 2500 & 2,125 \\
\hline 13 & María Auxiliadora - Derk & 1100 & 600 & 2500 & 2,125 \\
\hline 14 & Lubrigas & 1700 & 400 & 3000 & 2,550 \\
\hline 15 & Reimij & 1700 & 1000 & 3000 & 2,550 \\
\hline 16 & ATS América & 1700 & 1000 & 3000 & 2,550 \\
\hline 17 & Niyal & 1700 & 1000 & 3000 & 2,550 \\
\hline 18 & El Rosario & 1200 & 1000 & 2800 & 2,380 \\
\hline 19 & Vercia - El Chorrillano & 1100 & 2100 & 1800 & 1,530 \\
\hline 20 & Tablada & 1100 & 400 & 2500 & 2,125 \\
\hline 21 & Gessa & 1100 & 500 & 2500 & 2,125 \\
\hline 22 & Jevaro & 1700 & 1000 & 3000 & 2,550 \\
\hline 23 & San Borja - ACOSA & 1700 & 800 & 3000 & 2,550 \\
\hline 24 & Gasocentro Norte & 2000 & 1500 & 4500 & 3,825 \\
\hline 25 & Sol de Oro & 1500 & 1200 & 3500 & 2,975 \\
\hline 26 & Ultragrifos & 1700 & 500 & 3000 & 2,550 \\
\hline 27 & Collique & 1700 & 1000 & 3000 & 2,550 \\
\hline 28 & E/S Alta Gracia - Derk & 1100 & 400 & 2500 & 2,125 \\
\hline
\end{tabular}

\section{Cálculo del Stock de reposición}

Actualmente, la empresa dispone de camiones cisternas con capacidad de 5000 galones para el reparto de cada mañana, a sus diferentes unidades de atención; repartidos en todos los distritos de Lima, capital. En total son 27 grifos con capacidades nominales, en su mayoría entre 2500 y 3000 galones.

A partir de la ecuación contable:

Stock de Reposición = Capacidad real - Demanda

Se efectúa el cálculo del Stock de reposición, donde la demanda diaria es producto de las ventas de cada jornada; dato este, que cada fin del día, puede ser conocido de manera exacta. Obteniéndose el Stock de reposición desde el valor de la capacidad real. Se debe hacer notar, que la aplicación de esta ecuación nos es exacta, por los criterios de practicidad, por parte de los empleados, los que manejan cifras enteras, redondas a miles; lo que facilita, la labor de contabilizar la entrega resulte más simple. En la Tabla 2, también se presenta el cálculo del Stock de reposición para un día cualquiera.

\section{Cálculo de la distancia}

Con el conocimiento de la ubicación cartesiana de cada uno de los puntos en el plano de Lima (en este caso, grifos), se ha calculado utilizando la ecuación de la recta euclidiana para determinar la distan- 
cia entre cada par de grifos. Sea el grifo $i i$, en el punto $P_{i}=\left(x_{i}, y_{i}\right) P_{i}=\left(x_{i}, y_{i}\right)$ y el grifo $j j$ en el punto $P_{j}=\left(x_{j}, y_{j}\right) P_{j}=\left(x_{j}, y_{j}\right)$; la distancia euclidiana, se obtiene desde:

$$
d(i, j)=\sqrt[2]{\left(\left(x_{i}-x_{j}\right)^{2}+\left(y_{i}-y_{j}\right)^{2}\right)}
$$

En la Tabla 3, se presenta el valor de las distancias obtenidas para los diferentes pares de grifos $\left(27^{\star} 28 / 2=378\right)$. El punto Sede-origen es el depósito 1 o punto de inicio (orden 1).

Tabla 3. Distancia de los puntos de venta

\begin{tabular}{|c|c|c|c|}
\hline $\mathbf{N}$ & Desde & Hacia & Distancias $\mathbf{( k m )}$ \\
\hline 1 & Sede-origen & Sudamericano & 15 \\
\hline 2 & Sede-origen & Carmelo & 12 \\
\hline 3 & Sede-origen & Huiracocha & 33 \\
\hline 4 & Sede-origen & Monterrico & 38 \\
\hline 5 & Sede-origen & Canadá & 31 \\
\hline 6 & Sede-origen & Igarza & 9 \\
\hline 7 & Sede-origen & Quilca & 8 \\
\hline 8 & Sede-origen & Zarate & 21 \\
\hline 9 & Sede-origen & La Paz & 14 \\
\hline 10 & Sede-origen & La Marina - ACOSA & 21 \\
\hline 11 & Sede-origen & Ejercito - Acosa & 30 \\
\hline 12 & Sede-origen & María Auxiliadora - Derk & 17 \\
\hline 13 & Sede-origen & Lubrigas & 41 \\
\hline 14 & Sede-origen & Reimij & 27 \\
\hline 15 & Sede-origen & ATS America & 60 \\
\hline 16 & Sede-origen & Niyal & 25 \\
\hline 17 & Sede-origen & El Rosario & 39 \\
\hline 18 & Sede-origen & Vercia - El Chorrillano & 57 \\
\hline 19 & Sede-origen & Tablada & 58 \\
\hline 20 & Sede-origen & Jevaro & 63 \\
\hline 21 & Sede-origen & San Borja - ACOSA & 37 \\
\hline 22 & Sede-origen & Gasocentro Norte & 12 \\
\hline 23 & Sede-origen & Sol de Oro & 20 \\
\hline 24 & Sede-origen & Ultragrifos & 57 \\
\hline 25 & Sede-origen & Collique & 13 \\
\hline 26 & Sede-origen & E/S Alta Gracia - Derk & 16 \\
\hline 27 & Sede-origen & Gessa & 63 \\
\hline 28 & Depósito-2 & Carmelo & 8 \\
\hline 29 & Depósito-2 & Huiracocha & 10 \\
\hline 30 & Depósito-2 & Monterrico & \\
\hline
\end{tabular}

\section{Solución por metaheurística}

Existen diversas técnicas de búsqueda local con la finalidad de encontrar un mínimo local.

La búsqueda Tabú es una variante de la búsqueda local, una estrategia para resolver problemas de optimización combinatoria, donde se combina búsqueda local con una heurística para evitar parar en mínimos locales y evitar entrar en ciclos.

La búsqueda Tabú o Tabú Search (TS) es una metaheurística que guía un procedimiento de búsqueda heurística local para explorar el espacio más allá del óptimo local (10). Está basada en la premisa que un problema a ser resuelto es calificado como inteligente porque incorpora memoria adaptativa y exploración responsable.

La idea central radica en evitar un mínimo local, permitiendo movimientos que no mejoran la solución.

Según Fred Glover: "La TS incorpora elementos básicos combinando procedimientos híbridos con otras heurísticas y algoritmos que han tenido éxitos en hallar mejoras a la solución de los problemas" (11).

La evaluación TS empieza con una lista de candidatos a examinar. Se efectúa un test TS en el caso que la prueba traiga atributos que pertenecen al conjunto de atributos tabú, pasa a despenalizarlo; en otro caso, se aplica los criterios de aspiración.

En el algoritmo presentado en este trabajo, se recoge la aplicación de una serie de reglas para la obtención de la solución final de una manera aproximada. Estas reglas, se han integrado dentro de un algoritmo metaheurístico de solución, denominado CW3, en el que se utilizan reglas básicas para la construcción de las rutas. El objetivo es resolver el problema de forma aproximativa en un tiempo muy reducido de manera que la solución se devuelve posteriormente a la interfaz de la aplicación; desarrollada para la implementación del mismo, en el lenguaje JAVA. Los detalles del algoritmo y su aplicación se recogen en las siguientes líneas

El algoritmo del presente trabajo ha sido dividido en dos fases: la solución inicial y la fase de mejoramiento de la búsqueda local

\section{Fase de la solución inicial}

Un algoritmo VRP, normalmente comienza con una solución no factible, y en el pasaje de la búsqueda 
consigue soluciones factibles que en el ciclo de las iteraciones van mejorando la función objetivo.

Una simple lista de candidatos, puede constituir la denominada solución inicial. Primariamente la TS, construye una solución inicial, que al paso del test TS afina la búsqueda local. Como se desprende de Glover al definir TS, aquí se utilizan elementos básicos ya probados, en otras palabras, heurísticas que han tenido éxito en problemas similares, de allí que en vez de construir una solución inicial comúnmente no factible, se empiece con una solución factible.

\section{Fase de mejoramiento de la búsqueda local}

Se hace un análisis de las vecindades a la solución encontrada por CW. El procedimiento CW es una heurística probada, y se le une el test de exploración con la finalidad de crear un algoritmo híbrido que combina heurística con las reglas de la TS (13). En la segunda parte del algoritmo, se efectúa el mejoramiento a través de la metaheurística de los intercambios o Cross.

En resumen, el algoritmo aplicado a la empresa OLDR para la solución al VRP de sus servicentros resume dos probados y exitosos procedimientos lo que facilita grandemente reducir la parte computacional.

La primera fase del algoritmo es la generación de la lista inicial de candidatos a ser servidos por los vehículos cisternas; que recorrerán las $\boldsymbol{R}$ rutas. Esta primera lista se denomina $L_{1} L_{1}$, y contendrá exactamente a los $n$ nodos. La lista $L_{1}$, es resultado de aplicar el algoritmo heurístico de Clarke y Wright (CW). Para ello, el algoritmo en su fase 2 (6), se centra en el análisis de un conjunto de vecindades, desde $L_{1}$.

La aplicación del algoritmo CW, al problema de OLDR, genera una solución con una longitud total de $770 \mathrm{Km}$; longitud que consiste en la suma de los 11 tours, siendo la longitud de cada tour, medida entre todo el circuito desde que salen los cisternas del depósito 1 y su regreso.

En la tabla 4 se presenta la solución del algoritmo CW, donde se aprecia que, con capacidad de cisterna de 5000 galones; en algunas rutas, solo llevan 3400 galones con miras a minimizar la total distancia recorrida.
Tabla 4. Solución inicial con distancia 770

\begin{tabular}{|c|l|c|}
\hline Ruta & \multicolumn{1}{|c|}{ Tour } & Galones \\
\hline 1 & $1--20-21-28-25-1$ & 4800 \\
\hline 2 & $1-18-19-12-13-1$ & 4500 \\
\hline 3 & $1-5-14-3-1$ & 4500 \\
\hline 4 & $1-6-22-1$ & 3400 \\
\hline 5 & $1-15-16-1$ & 3400 \\
\hline 6 & $1-4-11-1$ & 3400 \\
\hline 7 & $1-9-17-1$ & 3400 \\
\hline 8 & $1-23-24-1$ & 3700 \\
\hline 9 & $1-2-10-1$ & 3400 \\
\hline 10 & $1-7-8-1$ & 3400 \\
\hline 11 & $1-26-27-1$ & 3400 \\
\hline
\end{tabular}

\section{Solución final}

Se considera el tipo de intercambio, denominado Cross (9) que generaliza los tres tipos de vecindad (Gendreau-Clarke). El nuevo tipo de vecindario se considera para el intercambio de cadenas de puntos (de tamaños $k$ y $k 1$ ) entre dos rutas diferentes.

En esta vecindad, se deduce que el tipo I es de 4-intercambios, el del tipo II es de 3-intercambios, y el del tipo III es de 2-intercambios. Generándose 3 vecindades, simbolizadas por $\mathbf{N 1}(x), \mathbf{N 2}(x), N 3(x)$ que corresponden a conjuntos de soluciones factibles.

La segunda fase del algoritmo CW3 (3, porque se basa en el intercambio de los 3 tipos), genera vecindades con la finalidad de encontrar soluciones factibles.

En la Tabla 5 se presenta la mejor solución alcanzada desde los diversos intercambios realizados en las vecindades.

Se observa que la distancia total cubierta es de 753, solución que es confirmada al ejecutar un programa entero usando el software LINGO. Esta solución de LINGO fue conseguida abortando la ejecución puesto que después de varias horas de ejecución el programa no había alcanzado de analizar el proceso de Branch \& Bond ( $B \& B)$, típico de estos programas de matrices generadoras, que hacen uso del proceso de los algoritmos en programación entera: el de Ramificar y Acotar (B\&B). 
Tabla 5. Solución final con distancia 753

\begin{tabular}{|c|l|c|}
\hline Ruta & \multicolumn{1}{|c|}{ Tour } & Galones \\
\hline 1 & $1-3-14-5-1$ & 4500 \\
\hline 2 & $1-6-9-1$ & 3400 \\
\hline 3 & $1-7-2-1$ & 3400 \\
\hline 4 & $1-8-16-1$ & 3400 \\
\hline 5 & $1-10-11-1$ & 3400 \\
\hline 6 & $1-12-19-25-18-1$ & 4900 \\
\hline 7 & $1-13-22-4-1$ & 4500 \\
\hline 8 & $1-17-15-1$ & 3400 \\
\hline 9 & $1-24-23-1$ & 3700 \\
\hline 10 & $1-27-1$ & 1700 \\
\hline 11 & $1-28-20-21-26-1$ & 5000 \\
\hline
\end{tabular}

Fuente: elaboración propia.

\section{CONCLUSIONES PRELIMINARES}

Las conclusiones obtenidas en el estudio detallado son las siguientes:

- Empleando la metodología propuesta en el presente estudio, se elaboró una propuesta de diseño de rutas óptimas, para el transporte de combustible a los grifos de la empresa OLDR en la ciudad de Lima.

- Dada la experiencia amplia de la empresa OLDR en el negocio de la venta de combustible, así como la capacidad de su fuerza laboral, no cuenta con ningún Sistema de Optimización de Distribución implementado en su organización siendo esto necesario para alcanzar los más altos estándares de calidad, medio ambiente y seguridad.

- Además los sobrecostos en el transporte, algunos días alcanzan el límite del $20 \%$, en todo el proceso de distribución, por consecuencia de falta de diseño de rutas óptimas.

- Permite una herramienta de soporte a la decisión de uso diario para la planificación de las operaciones en la entrega de combustible a los clientes.

- Reduce los altos costos de planificación de la ruta, que incluye el uso de horas extras en el personal planificador.

- Permite realizar un análisis de sensibilidad en el volumen de entrega, en la búsqueda de configuraciones de rutas alternativas, sin sacrificar los costos totales.

- La metaheurística con aplicación a la logística de distribución en un nuevo algoritmo, para la resolución del problema de planificación de rutas de transporte para delivery. Este problema se conoce en la literatura como el VRP o Vehicle Routing Problem.

- La administración de la cadena de suministros tiene consecuencias estratégicas porque sistema puede usarse para satisfacer prioridades competitivas (18), implica la coordinación de funciones clave de la empresa, como marketing, finanzas, ingeniería, sistemas de información, operaciones y creación de inventarios creando fortalezas competitivas en las organizaciones (1).

- El flujo de información electrónica ha transferido la información rutinaria al área de ventas permitiéndole acceso directo a los datos del punto de ventas y permitiendo la proyección de ventas y la programación de entregas de combustible según los requerimientos de la demanda.

- La estrategia pura del planeamiento agregado consideró la variación de la mano de obra, tiempo extra, tiempo ocioso, variación de los niveles de inventario, reprocesos, subcontrataciones, utilización de capacidad y las variables de contingencias reales e impredecibles de la ciudad de lima.

- Aplicación de un algoritmo metaheurístico de solución con el objetivo de resolver el problema en forma aproximativa en un tiempo muy reducido, de manera que la solución se devuelve posteriormente a la interfaz de la aplicación; desarrollada para la implementación del mismo en el lenguaje JAVA.

El problema de recurrir a procedimientos "inteligentes" es por el recurso computacional. Las metaheurísticas han ganado aceptación por el reducido tiempo que utilizan en la búsqueda de un mejoramiento de la solución.

\section{REFERENCIAS BIBLIOGRÁFICAS}

[1] Acevedo y Cachay (2010). Gestión de operaciones y cadena de suministro. Planeamiento, programación y control de operaciones y logística. Primera edición, Ed. UNMSM, Perú.

[2] Ahuja, Ravindran, Magnanti, Orlin (2003). Network Flows Theory Algorithms and Applications. Ed. Prentice-Hall, Inc., USA.

[3] Bradley, Stephen, Cox, Magnanti (1977). Applied Mathematical Programming. Ed. Addison-Wesley Publishing Company, USA. 
[4] Glover, Fred (2003). "Handbook of metaheuristics", en Kluwer International Series, Kluwer Academic Publishers, USA.

[5] Ibaraki, Toshihide (2005). "Metaheuristics: Progress as Real Problem Solvers", en Interfaces Series, Springer Science + Business Media, Inc., USA.

[6] Clarke y Wright (1964). "Scheduling of vehicles from a central depot to a number of delivery points", en Operations Research Review $n^{\circ} 12$.

[7] Dantzig y Ramser (1959). "The truck dispatching problem", en Management Sciences Review $\mathrm{N}^{\circ} 6$, USA.

[8] Dorigo, Marcos (1997). "Ant Colony System: A Cooperative learning Approach to the Traveling Salesman problem", en IEEE Transactions on Evolucionary Computation, Vol. I, N0. 1, April 1997.

[9] Gendreau, Hertz, Laporte (1994). "A tabu Search heuristic for the vehicle routing problem", en Management Sciences 40.

[10] Glover, Fred (1989). "Tabu Search, Part 1", ORSA J. Computing No 1.

[11] Glover, Klingman, Phillips (1992). Network Models in Optimization and Their Applications in Practice. Ed. John Wiley \& Sons, Inc., USA.
[12] Goldberg, David E. (1989). "Genetic Algorithms in Search, Optimization and Machine Learning". Addison-Wesley Publishing Company, Inc.

[13] Hertz, de Werra (1987). "Using Tabu Search Techniques for Graph Coloring”, en Computing 39.

[14] Johnson, Aragón, McGeoch (1991). "Optimization by Simulated Annealing: an Experimental Evaluation: Part II, Graph Coloring and Number Partitioning", Operations Research, Vol. 39, No. 3, May-June 1991.

[15] Laporte y Norbert 1987). "Exact algorithms for the vehicle routing problem", en Annals of Discrete Mathematics 31, 1987.

[16] Michalewicz, Zbigniew (1996). “Genetic Algorithms+Data Structures=Evolution Programs", Springer-Verlag Berlin Heidelberg, Germany.

[17] Toth y Vigo, "An Overview of Vehicle Routing Problems", en Monographs on Discrete Mathematics and Applications. In: The Vehicle Routing Problems. SIAM 2000.

[18] Viitt, Elizabeth (2003). Business Intelligence. Ed. Mcgraw-Hill, España. 